\title{
Biological function of eosinophil extracellular traps in patients with severe eosinophilic asthma
}

\author{
Youngwoo Choi ${ }^{1}$, Duy Le Pham ${ }^{1,2}$, Dong-Hyun Lee ${ }^{1}$, So-Hee Lee', Seung-Hyun Kim (i) $^{3}$ and Hae-Sim Park ${ }^{1,3}$
}

\begin{abstract}
Eosinophil extracellular traps (EETs), a complex of DNA fibers and cytotoxic granule proteins, are implicated in the development of asthma; however, the pathophysiological function of EETs in immune responses has not been fully determined. The present study investigated the characteristics of EETs from patients with non-severe asthma (NSA, $n=20)$ and severe eosinophilic asthma (SEA, $n=20$ ) and evaluated EET function. The percentage of EET-forming peripheral blood eosinophils stimulated with IL-5 and LPS was significantly higher in patients with SEA than in those with NSA $(P=0.009)$. This percentage negatively correlated with baseline FEV $V_{1}(r=-0.350, P=0.027)$ and positively correlated with serum eosinophil-derived neurotoxin levels in asthmatic subjects $(r=0.437, P=0.018)$. In addition, EET formation was markedly associated with reactive oxygen species production $(r=0.750, P<0.001)$. These EETs exhibited an autocrine function to induce eosinophil degranulation, which led to granule protein production. Airway epithelial cells stimulated with EETs exhibited increased epithelial detachment and permeability and pro-inflammatory cytokine release. However, EETs were not significantly associated with mast cell activation. The present study suggests that peripheral blood eosinophils from patients with SEA may be more activated to produce EETs than those from patients with NSA, which further induces inflammation in asthmatic airways. Therefore, regulation of EET formation and function may be a novel therapeutic approach for asthma management.
\end{abstract}

\section{Introduction}

Asthma is a complicated airway disease with bronchial hyperresponsiveness ${ }^{1}$. The disease exists worldwide in a broad spectrum of phenotypes, and 300 million people are affected. However, $10 \%$ of asthmatic patients with severe symptoms are poorly controlled with conventional treatment $^{2,3}$. These patients experience frequent asthma exacerbations with morbidity/mortality and consume more healthcare resources, which results in hospital admissions and work loss ${ }^{4}$. Several studies investigated the pathophysiology of severe eosinophilic asthma (SEA),

\footnotetext{
Correspondence: Hae-Sim Park (hspark@ajou.ac.kr)

${ }^{1}$ Department of Allergy and Clinical Immunology, Ajou University School of

Medicine, Suwon, South Korea

${ }^{2}$ Faculty of Medicine, University of Medicine and Pharmacy, Ho Chi Minh,

Vietnam

Full list of author information is available at the end of the article.

These authors contributed equally: Youngwoo Choi, Duy Le Pham.
}

but a distinct immunological pathway was not fully determined because asthma is highly heterogeneous.

Eosinophilic inflammation is a key feature of asthma severity because it generates lung dysfunction ${ }^{5,6}$. Eosinophils play an important role in innate immunity during parasitic and helminthic infections ${ }^{7}$. However, whether these cells are beneficial in asthma is not certain because substantial evidence has demonstrated a role for eosinophils in tissue damage and asthma symptoms ${ }^{8}$. Eosinophilia generates airway inflammation and remodeling, which are closely associated with asthma exacerbation in $\mathrm{SEA}^{9-11}$. Eosinophil-derived granule proteins involved in immune responses, such as major basic protein, eosinophil cationic protein (ECP) and eosinophil-derived neurotoxin (EDN), were extensively studied ${ }^{12-14}$. High levels of granule proteins are indicative of eosinophil activation and degranulation, which is associated with SEA. 
Eosinophil extracellular traps (EETs) are a complex meshwork of DNA fibers and granule proteins that play a role in innate immunity to infectious disease and induce tissue damage in asthmatic airways ${ }^{15-18}$. Eosinophils release EETs in a NADPH oxidase-dependent manner associated with reactive oxygen species (ROS) production $^{19-21}$. Neutrophils also produce extracellular traps via rapid cell death ${ }^{22,23}$. The pathogenic or harmful effect of an excess of neutrophil extracellular traps (NETs) was investigated ${ }^{24,25}$. NETs are present in multiple diseases, including asthma ${ }^{26,27}$. However, whether EETs contribute to the pathogenesis of asthma because of unique biological activities is not known.

The present study hypothesized that more activated peripheral blood eosinophils contribute to higher levels of EET formation, which leads to enhanced airway inflammation in asthmatic patients, especially SEA. Therefore, the effects of EETs on major inflammatory cells and airway epithelial cells involved in immune responses were evaluated.

\section{Materials and methods Study subjects}

The Institutional Review Board of Ajou University Hospital approved this study (AJIRB-GEN-SMP-13-108). All study subjects provided written informed consent at the time of recruitment. We enrolled 20 patients with SEA and 20 patients with non-severe asthma (NSA). All patients were diagnosed based on histories of recurrent episodes of wheezing, dyspnea, cough, sputum production, and evidence of airway hyperresponsiveness to methacholine or reversible airway obstruction that improved with a short-acting $\beta_{2}$ agonist $^{28}$. Levels of ECP and EDN in serum were measured using the enzymelinked immunosorbent assays (ELISA) kits (MBL, Sakae, Japan), according to the manufacturer's instructions. All patients enrolled in this study had a history of asthma exacerbations that required systemic steroids (higher than $15 \mathrm{mg}$ or prednisolone/d for $>3 \mathrm{~d}$ ) and sputum eosinophilia (greater than $3 \%$ of sputum leukocytes counted). Patients who were current smokers, exhibited comorbidity with chronic obstructive pulmonary disease or other chronic diseases affecting asthma outcomes were excluded. The SEA group was defined, as described previously $^{29}$.

\section{Isolation of peripheral blood eosinophils}

Venous blood was collected from study subjects in stable, not exacerbation, states. Samples were collected in BD Vacutainer tubes containing acid citrate dextrose solution (BD Biosciences, Franklin Lakes, NJ, USA), stored at room temperature, and processed within $2 \mathrm{~h}$ of collection. Blood was layered on a Lymphoprep ${ }^{\mathrm{m}}$ Solution (Axis-Shield, Oslo, Norway), followed by centrifugation at
$879 \times g$ at $20^{\circ} \mathrm{C}$ for $25 \mathrm{~min}$ without braking. Eosinophils were isolated from the fraction containing red blood cells and granulocytes using the Eosinophil Isolation Kit and MACS Column (Miltenyi Biotec Inc., Auburn, CA, USA) according to the manufacturer's instructions.

\section{Isolation of eosinophil extracellular traps}

Peripheral blood eosinophils $\left(1 \times 10^{6}\right)$ were primed with $25 \mathrm{ng} / \mathrm{mL}$ human recombinant IL-5 (Sigma-Aldrich, St. Louis, MO, USA) for $20 \mathrm{~min}$ and stimulated with $0.3 \mu \mathrm{g} / \mathrm{mL}$ LPS (Sigma-Aldrich) for $3 \mathrm{~h}$ to induce EETs. DNA concentration was measured using the Quant-iT ${ }^{\mathrm{min}}$ PicoGreen ${ }^{\circ}$ dsDNA kit (Invitrogen, Paisley, UK). Protein concentrations were evaluated using the QuantiPro BCA Assay Kit (Sigma-Aldrich). Cells were stained with 4',6-diamidino-2phenylindole and anti-EPO antibody (Cell Signaling, Minneapolis, MN, USA) and analyzed using a Zeiss LSM710 confocal microscope (Carl Zeiss AG, Oberkochen, Germany) to detect EET-forming eosinophils.

\section{Treatment with dexamethasone, hydroxychloroquine, or antioxidant}

Peripheral blood eosinophils from patients with NSA were maintained in serum-free RPMI-1640 medium (Thermo Fisher Scientific, Waltham, CA, USA) and pretreated with $1 \mu \mathrm{M}$ dexamethasone (Sigma-Aldrich), 10 $\mu \mathrm{M}$ hydroxychloroquine (Sigma-Aldrich), or $1 \mu \mathrm{g} / \mathrm{mL} \mathrm{N}$ Acetyl-L-cysteine (Sigma-Aldrich) for $15 \mathrm{~min}$ prior to IL-5 and LPS stimulation. Cells were treated with $10 \mu \mathrm{M}$ $\mathrm{H}_{2}$ DCFDA (Thermo Fisher Scientific) and incubated for $30 \mathrm{~min}$ at $37^{\circ} \mathrm{C}$ to detect intracellular reactive oxygen species. Propidium iodide $(1 \mu \mathrm{g} / \mathrm{mL}$; Thermo Fisher Scientific) was added, and signals were read at a wavelength of $620 \mathrm{~nm}$.

\section{Stimulation of airway epithelial cells}

A549 cells, BEAS-2B cells, and human primary small airway epithelial cells (American Type Culture Collection, Manassas, VA, USA) were purchased. A549 cells and BEAS-2B cells were cultured in RPMI-1640 medium supplemented with $10 \%$ fetal bovine serum, $100 \mathrm{IU} / \mathrm{mL}$ penicillin and $50 \mu \mathrm{g} / \mathrm{mL}$ streptomycin (all components from Thermo Fisher Scientific). Human primary small airway epithelial cells were cultured in airway epithelial cell basal medium supplemented with $2.4 \mathrm{mM}$ L-alanyl-L glutamine, $0.4 \%$ extract $\mathrm{P}, 0.5 \%$ plasma protein fraction, $1.0 \mu \mathrm{M}$ epinephrine, $5 \mu \mathrm{g} / \mathrm{mL}$ transferrin, $10 \mathrm{nM} \mathrm{T} 3,5 \mu \mathrm{g} /$ $\mathrm{mL}$ hydrocortisone, $5 \mathrm{ng} / \mathrm{mL}$ human recombinant epidermal growth factor, and $5 \mu \mathrm{g} / \mathrm{mL}$ human recombinant insulin (all components were provided in a Small Airway Epithelial Cell Growth Kit, ATCC). Cells were treated with $10 \mathrm{nM}$ phorbol myristate acetate (Sigma-Aldrich) or $5 \mu \mathrm{g} / \mathrm{mL}$ EETs for $24 \mathrm{~h}$. IL-8 (Endogen, Woburn, MA, USA) and IL-6 (R\&D Systems, Minneapolis, MN, USA) 
concentrations in culture supernatants were measured using ELISA kits according to the manufacturer's protocol.

\section{Evaluation of cell detachment}

Cell numbers were assessed using a cell counting kit (CCK)-8 (Dojindo Molecular Technologies, Kumamoto, Japan) according to the manufacturer's protocol. Culture plates were washed once with PBS to eliminate detached cells prior to addition of the CCK-8 solution and evaluate cell detachment. The \%cell detachment $=[$ (number of cells in the unwashed plates-number of cells in the washed plate)/number of cells in unwashed plates] $\times$ $100 \%$.

\section{Epithelial permeability assay}

A549 cells were cultured on collagen-coated transwell chambers and treated with EETs for $24 \mathrm{~h}$. The apical chamber was washed with Hank's balanced salt solution, and $1 \mathrm{mg} / \mathrm{mL}$ FITC-dextran (Sigma-Aldrich) was added for $6 \mathrm{~h}$. Media from the basal chamber were collected, and fluorescence intensity was measured (excitation, $490 \mathrm{~nm}$ and emission, $530 \mathrm{~nm}$ ).

\section{$\beta$-hexosaminidase release test}

LAD-2 cells (National Institute of Allergy and Infectious Diseases, Bethesda, MD, USA) were maintained in StemPro-34 medium (Life Technologies, Grand Island, NY, USA) supplemented with $2 \mathrm{mM}$ L-glutamine, $100 \mathrm{U} /$ $\mathrm{mL}$ penicillin, $50 \mu \mathrm{g} / \mathrm{mL}$ streptomycin, and $100 \mathrm{ng} / \mathrm{mL}$ recombinant human stem cell factor (R\&D Systems). LAD-2 cells were sensitized using $100 \mathrm{ng} / \mathrm{mL}$ biotinylated-IgE (BioPorto Diagnostics, Hellerup, Denmark) with or without $5 \mu \mathrm{g} / \mathrm{mL}$ EETs. Cells were stimulated with $100 \mathrm{ng} / \mathrm{mL}$ streptavidin peroxidase (Sigma-Aldrich) in Tyrode's buffer containing 0.1\% BSA. Total $\beta$-hexosaminidase was obtained via lysing of LAD-2 cells in $0.1 \%$ Triton X-100 in PBS. The supernatants were collected and incubated with an equal volume of $4 \mathrm{mM}$ $p$-nitrophenyl $N$-acetyl- $\beta$-D-glucosamide (Sigma-Aldrich) in citrate buffer for $1 \mathrm{~h}$. The reactions were stopped via the addition of $0.4 \mathrm{M}$ glycine buffer, and signals were read at a wavelength of $405 \mathrm{~nm}$. Levels of TNF- $\alpha$ and MCP-1 (R\&D Systems) were measured using ELISA kits according to the manufacturer's protocol.

\section{Statistical analysis}

Student's $t$ test was used to compare continuous variables, and Pearson's chi-square test was used for categorical variables. Statistical correlations were analyzed using Pearson's rank coefficient. All statistical analyses were performed using IBM SPSS software version 22.0 (IBM Corp., Armonk, NY, USA). $P$ values $<0.05$ were considered to indicate statistical significance. GraphPad
Prism 5.0 software (GraphPad Inc., San Diego, CA, USA) was used to create graphs.

\section{Results}

\section{Demographic data from study subjects}

Table 1 shows the clinical characteristics of the study subjects. Mean baseline $\mathrm{FEV}_{1}$ and $\mathrm{PC}_{20}$ methacholine levels were significantly lower in the SEA group than in the NSA group $(P=0.001, P=0.013)$. Sputum eosinophils (\%) and serum EDN levels were markedly higher in patients with SEA than in those with NSA $(P=0.024, P=$ 0.005). Serum ECP levels tended to be higher in SEA.

\section{Characterization of EETs from asthmatic patients}

Peripheral blood eosinophils were treated with IL-5 and LPS to investigate EET formation. The percentage of EET-forming eosinophils was measured using immunostaining (Fig. 1a). Eosinophils were stimulated, and the proportion of cells releasing EETs was significantly higher in the SEA group than in the NSA group. However, unstimulated eosinophils exhibited no significant differences between the 2 groups (Fig. 1b), which indicates that eosinophils from patients with SEA may be more easily activated to produce EETs. The relationship of EETforming eosinophils with clinical parameters in asthma was investigated. A negative correlation between EET formation and baseline $\mathrm{FEV}_{1} \%$ predicted values was observed ( $r=-0.350, P=0.027$; Fig. 1c), and a positive correlation was found between EET formation and serum EDN levels ( $r=0.437, P=0.018$; Fig. $1 d)$.

Table 1 Clinical characteristics of the study subjects

\begin{tabular}{|c|c|c|c|}
\hline Variables & NSA $(n=20)$ & $\operatorname{SEA}(n=20)$ & $P$ value \\
\hline Age (year) & $54.9 \pm 15.6$ & $57.5 \pm 6.5$ & 0.506 \\
\hline Sex (Male, \%) & 50.0 & 30.0 & 0.333 \\
\hline Atopy (\%) & 60.0 & 65.0 & 1.000 \\
\hline Baseline $\mathrm{FEV}_{1}(\%)$ & $100.1 \pm 10.9$ & $72.5 \pm 15.9$ & 0.001 \\
\hline $\mathrm{PC}_{20}$, methacholine $(\mathrm{mg} / \mathrm{mL})$ & $12.9 \pm 10.8$ & $2.5 \pm 1.4$ & 0.013 \\
\hline Serum total lgE (IU/ml) & $302.3 \pm 334.4$ & $249.3 \pm 184.8$ & 0.540 \\
\hline Total eosinophil count $(/ \mu \mathrm{L})$ & $380.1 \pm 661.8$ & $389.0 \pm 399.2$ & 0.959 \\
\hline Sputum eosinophils (\%) & $16.1 \pm 26.9$ & $40.9 \pm 38.7$ & 0.024 \\
\hline Sputum neutrophils (\%) & $67.3 \pm 30.5$ & $48.2 \pm 36.7$ & 0.082 \\
\hline Serum ECP (ng/mL) & $74.5 \pm 56.5$ & $93.9 \pm 68.6$ & 0.398 \\
\hline Serum EDN (ng/mL) & $28.5 \pm 12.2$ & $44.9 \pm 16.4$ & 0.005 \\
\hline
\end{tabular}

Values are given as $n(\%)$ for categorical variables and as the mean \pm SD for continuous variables

$P$ values were calculated using the Pearson chi-square test for categorical variables and Student's $t$ test for continuous variables

$F E V_{1}$ forced exhaled volume at $1 \mathrm{~s}, P C_{20}$ concentration of methacholine to induce a $20 \%$ decline in $\mathrm{FEV}_{1}, E C P$ eosinophil cationic protein, $E D N$ eosinophil-derived neurotoxin, NSA non-severe asthma, SEA severe eosinophilic asthma

Bold values indicate the clinical variables which show statistical significance 

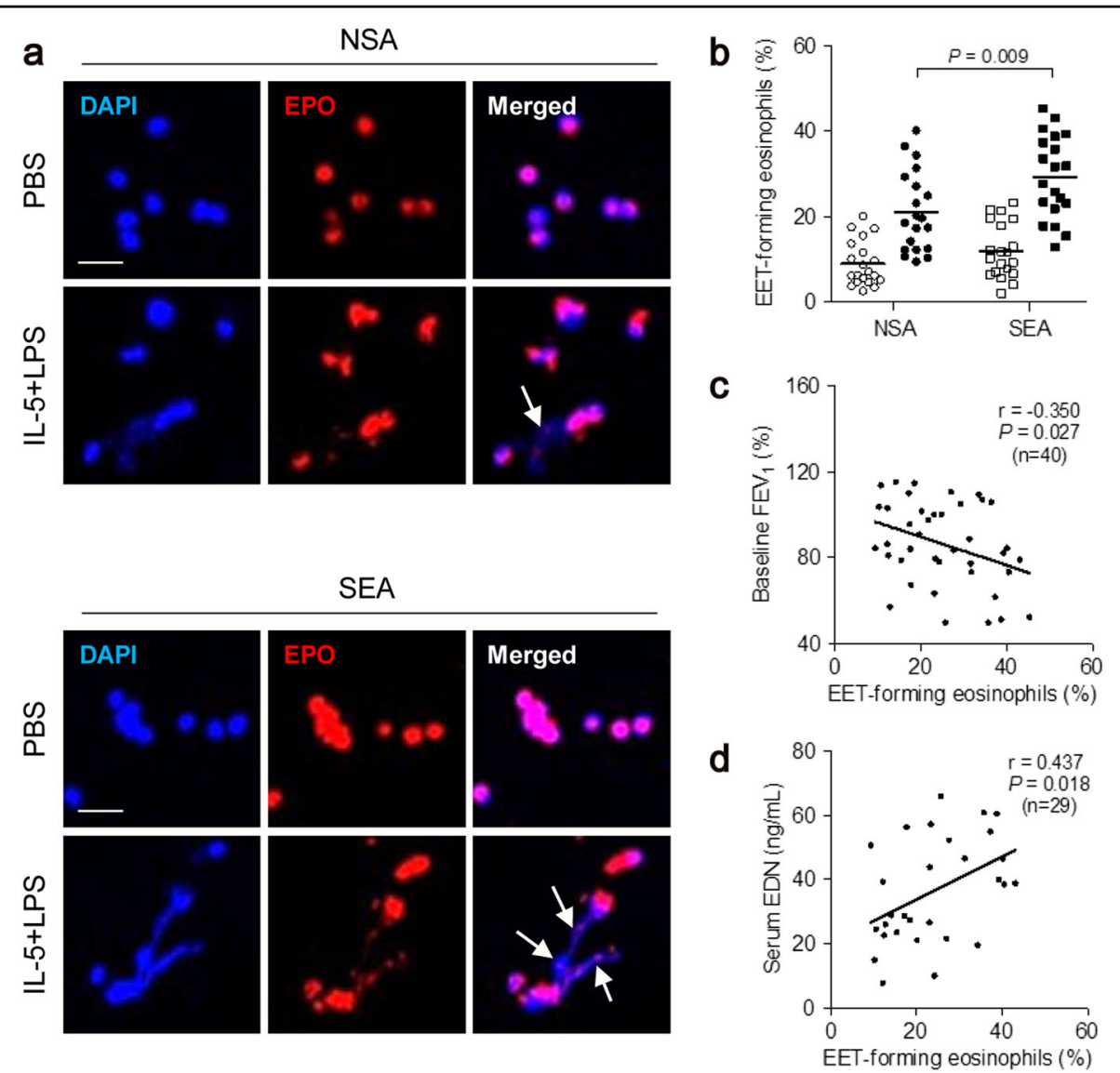

Fig. 1 Characteristics of EETs from peripheral blood eosinophils of patients with NSA and SEA. a Induction of EET formation (white arrows). Scale bar, $50 \mu \mathrm{m}$. b Comparison of the percentage of EET-forming eosinophils with (closed) or without (open) IL-5 and LPS stimulation. Associations between EET formation and $\mathbf{c}$ baseline $\mathrm{FEV}_{1} / \mathbf{d}$ serum EDN levels. The data are presented as Pearson correlation coefficient $r(P$ value)

Association between EET formation and ROS production

Peripheral blood eosinophils from patients with NSA were treated with various drugs to investigate the regulation of EET formation via inhibition of ROS production. $N$-acetyl-L-cysteine (an antioxidant) markedly reduced the percentage of EET-forming eosinophils stimulated with IL-5 and LPS. Dexamethasone (a representative anti-inflammatory drug used in the treatment of asthma) and hydroxychloroquine (an autophagy inhibitor) produced no significant effect (Fig. 2a). N-acetyl-Lcysteine, but not hydroxychloroquine, also attenuated ROS production (Fig. 2b). EET formation was positively correlated with ROS production $(r=0.750, P<0.001$; Fig. 2c), which indicates that regulation of ROS production effectively controls EET-forming eosinophils.

\section{Autocrine function of EETs in eosinophil activation}

The current study investigated whether EETs enhanced eosinophilic inflammation via formation of a vicious cycle with autocrine function. Peripheral blood eosinophils were treated with EETs, and the effect of EETs was compared with that of phorbol myristate acetate (PMA), which is a strong stimulator of eosinophils. PMA and EETs produced morphological changes in eosinophils, which exhibited a hole on the cell surface (Fig. 3a). Confocal images revealed that EETs generated EET formation from eosinophils themselves (Fig. 3b). EET treatment also significantly elevated cell degranulation and ROS production. However, the effect was weaker for EET treatment than for PMA treatment (Fig. 3c, d). The present study also evaluated the effects of dexamethasone, hydroxychloroquine or $N$-acetyl-L-cysteine on EETtreated eosinophils. Dexamethasone and $N$-acetyl-Lcysteine attenuated the production of granule proteins (Fig. 3e).

\section{EET-induced airway epithelial cell detachment and immune responses}

EETs altered the morphology and density of A549 cells in a dose-dependent manner (Fig. 4a). EETs induced $>10 \%$ cell detachment (Supplementary Figure 1a). Airway epithelial cells were cultured in transwells, and the 

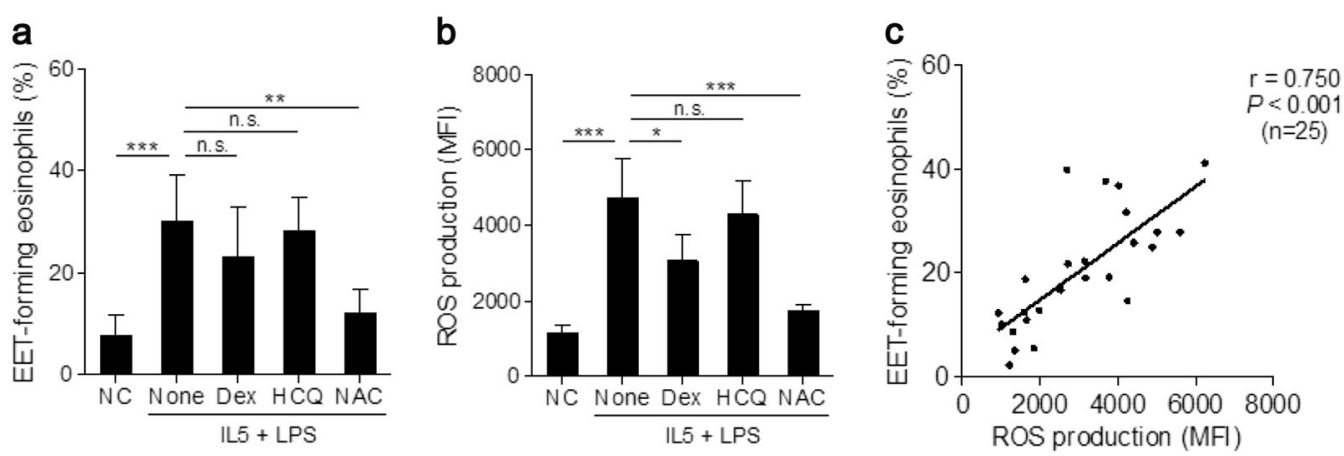

Fig. 2 Reactive oxygen species (ROS)-dependent EET production from peripheral blood eosinophils of patients with NSA. Effects of dexamethasone (Dex), hydroxychloroquine (HCQ) or N-acetyl-L-cysteine (NAC) on a EET formation and $\mathbf{b}$ ROS production. Data are presented as the mean $\pm \mathrm{SD}, n=5 .{ }^{*} P<0.05,{ }^{*} P<0.01$ and ${ }^{* *} P<0.001$ obtained using one-way ANOVA with Bonferroni's post hoc test. n.S., not significant. $\mathbf{c} A$ correlation between ROS and EET production. The data are presented as Pearson correlation coefficient $r(P$ value)
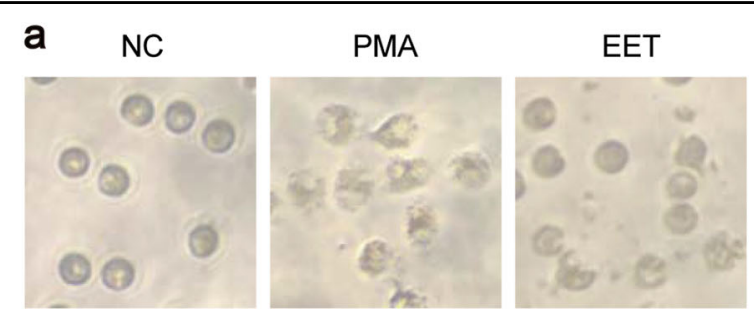

C

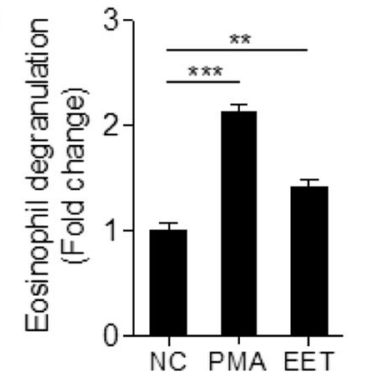

b
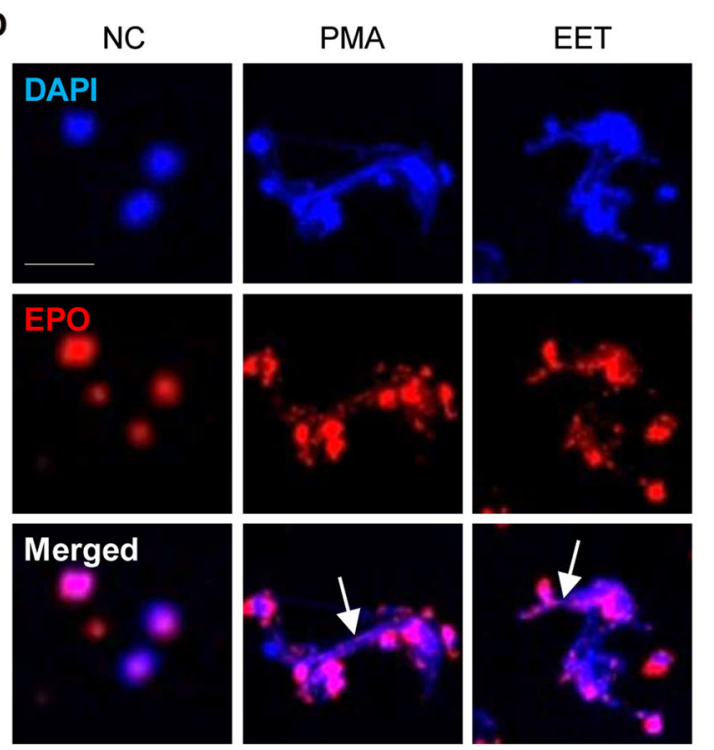

d

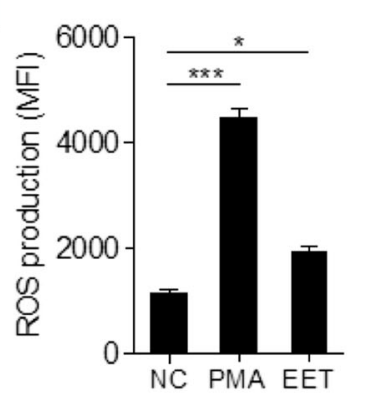

e

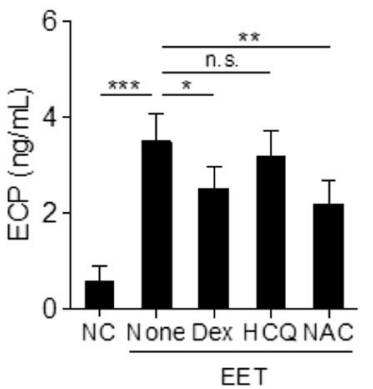

Fig. 3 Autocrine function of EETs on eosinophil activation. a Morphological changes of peripheral blood eosinophils from patients with NSA after $100 \mathrm{nM}$ phorbol myristate acetate (PMA) or $5 \mu \mathrm{g} / \mathrm{mL}$ EET (from NSA) treatment. $\mathbf{b}$ Immunofluorescence staining of released EETs (white arrows). Scale bar, $10 \mu \mathrm{m}$. c Eosinophil degranulation. d ROS production. e Effect of Dex, HCQ and NAC on eosinophil production of ECP. Data are presented as the mean, $n=5$. ${ }^{*} P<0.05,{ }^{* *} P<0.01$, and ${ }^{* *} P<0.001$ obtained using one-way ANOVA with Bonferroni's post hoc test. n.s., not significant 

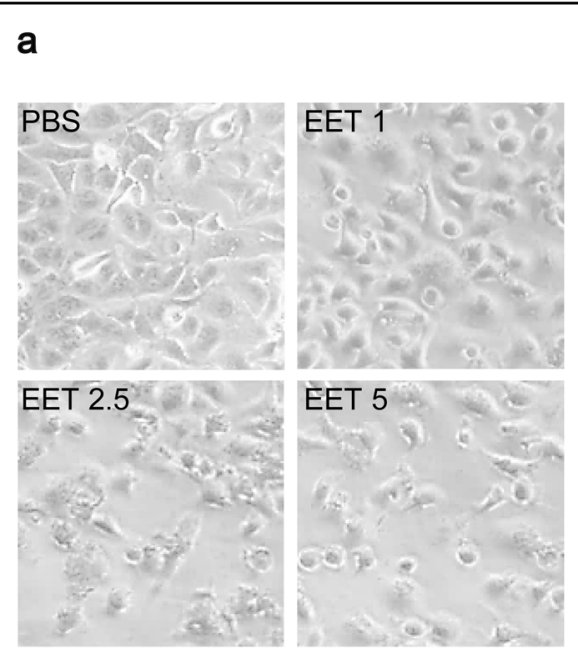

b
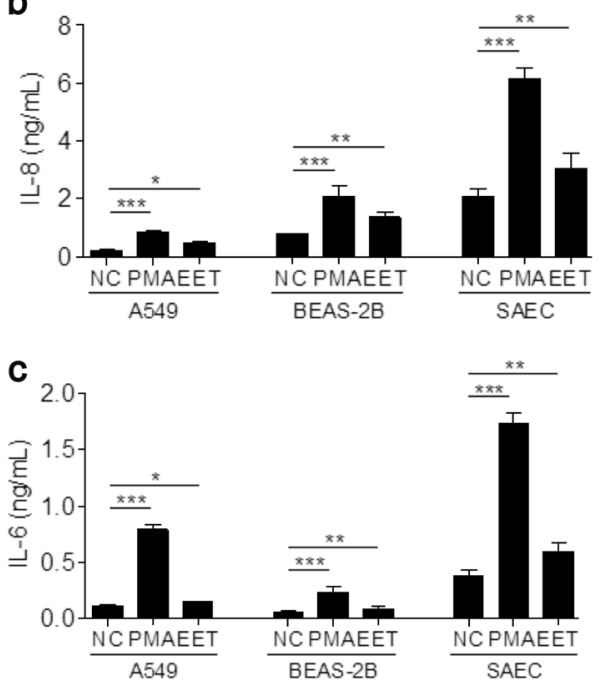

Fig. 4 Effect of EETs on airway epithelial cells. a Changes in A549 cell morphology following EET (from NSA) treatment in a dose-dependent manner. Concentrations of $\mathbf{b} \| \mathrm{L}-8$ and $\mathbf{c} \| \mathrm{L}-6$ released from A549, BEAS-2B, and human primary small airway epithelial cells (SAEC) treated with $5 \mu \mathrm{g} /$ $\mathrm{mL}$ EETs. Data are presented as the mean $\pm \mathrm{SD}, n=5$. ${ }^{*} P<0.05,{ }^{* *} P<0.01$, and ${ }^{* * *} P<0.001$ were obtained using one-way ANOVA with Bonferroni's post hoc test

fluorescence intensity of FITC-dextran in the basal chamber was measured to confirm the effect of EETs on epithelial permeability. EET-treated A549 cells exhibited greater fluorescence than untreated cells, which indicates that EETs increased epithelial permeability (Supplementary Figure 1b). The present study evaluated the capacity of EETs to induce pro-inflammatory cytokine production from airway epithelial cells. EETs significantly enhanced IL-8 and IL-6 release from A549, BEAS-2B and human primary small airway epithelial cells (Fig. 4b, c). Dexamethasone, an anti-MBP antibody and anti-ECP antibody were used to determine whether EET-mediated immune responses could be regulated. Dexamethasone and the anti-ECP antibody, but not the anti-MBP antibody, strongly reduced the production of IL-8 from A549 cells (Supplementary Figure 2). These data suggest that cytotoxic granule proteins that are co-localized in EETs are an important factor in inducing airway inflammation from epithelial cells.

\section{Effect of EETs on mast cell activation}

LAD-2 cells were treated with EETs in the presence or absence of IgE to evaluate the effect of EETs on mast cell activation. LAD-2 cells stimulated with EETs exhibited no significant differences in cell degranulation compared with controls regardless of the presence of IgE (Fig. 5a), which indicates that EETs do not play a crucial role in the induction of mast cell degranulation. EET treatment did not markedly enhance TNF- $\alpha$ or MCP-1 release from LAD-2 cells (Fig. 5b).

\section{Discussion}

This study is the first report to demonstrate a harmful effect of eosinophils via EET production with an autocrine function and induction of immune responses in asthma. Eosinophils from patients with SEA were more activated than eosinophils from patients with NSA, which increased ROS production and EET formation. In addition, the percentage of EET-forming eosinophils was negatively correlated with baseline $\mathrm{FEV}_{1}$. These findings suggest that EETs play a critical role in eosinophilic airway inflammation.

Several previous papers demonstrated the presence of EETs in multiple eosinophilic diseases ${ }^{15-17}$. A previous paper suggested enhanced EET formation in the airway mucosa of asthmatic patients with severe airway inflammation ${ }^{18}$. The present study revealed that EET-forming eosinophils were negatively correlated with baseline $\mathrm{FEV}_{1}$ $\%$ predicted values and positively correlated with serum EDN levels in asthmatic patients. Peripheral blood eosinophils isolated from patients with SEA exhibited increased EET production following activation with IL-5 and LPS in an ROS-dependent manner. Our results suggest that increased EETs are closely associated with airway inflammation and obstruction in patients with SEA.

Airway epithelial cells are the first-line barrier against antigens in the lungs ${ }^{30}$. These cells are an essential immunomodulator of immune responses to the allergens, viruses and pollutants involved in the pathogenesis of asthma ${ }^{31}$. The present study demonstrated that EETs contributed to the impairment of airway integrity via 

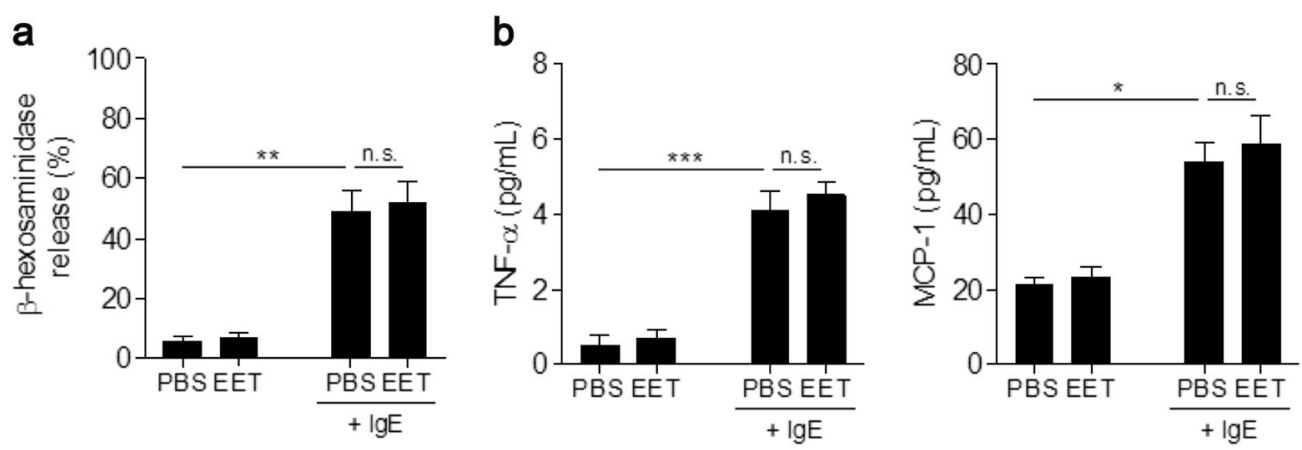

Fig. 5 Effect of EETs on mast cell activation. a LAD-2 cell degranulation following EET (from NSA) treatment. $\mathbf{b}$ Levels of TNF-a and MCP-1 released from LAD-2 cells. Data are presented as the mean $\pm S D, n=3$. ${ }^{* *} P<0.01$ was obtained using one-way ANOVA with Bonferroni's post hoc test. n.S., not significant

airway epithelial detachment, which increased epithelial permeability and enhanced airway inflammation in asthmatic airways. Cell death follows cell detachment because airway epithelial cells require adhesion to the basement membrane for survival ${ }^{32}$. We confirmed the proinflammatory effect of EETs using IL-8 production from airway epithelial cells. Increased IL-8 concentrations are commonly found in inflammatory sites in patients with respiratory virus infection, chronic obstructive pulmonary diseases or asthma ${ }^{33}$. Many previous studies focused on the inhibition of eosinophil-derived cytotoxic granule proteins, such as MBP and ECP, to regulate immune responses in the airway epithelium ${ }^{34}$. Our data demonstrated that a specific antibody against ECP significantly reduced EET-mediated pro-inflammatory cytokine production from airway epithelial cells. Dexamethasone also significantly decreased IL-8 production, but this result may be a simple anti-inflammatory effect on these cells. These findings suggest that further investigational drugs are needed to suppress the EET-epithelial cell axis in patients with SEA.

Mast cells contribute to the pathogenesis of SEA. Mast cells produce histamine and type 2 cytokines, such as IL4, IL-5, and IL-13, which regulate the development and activation of eosinophilic inflammation ${ }^{35}$. Mast cells play a crucial role in asthma-related airway inflammation, and the present study investigated the effect of EETs on mast cells. However, EETs did not significantly induce degranulation or cytokine production; hence, IgE may be more important for mast cell stimulation.

The current study has some limitations. First, the pathophysiological function of EETs was not confirmed in vivo. A previous study revealed elevated EET formation in ovalbumin-challenged mice, but the effect of EETs in eosinophilic asthma is not clear ${ }^{36}$. Second, EET-forming eosinophils in the lungs of asthmatic patients were not investigated, which would provide direct evidence for EETs in the pathogenesis of asthma. Further investigations of the detailed underlying molecular mechanisms and new potential therapeutic targets are warranted.

In conclusion, EETs may play an important role in the pathogenesis of eosinophilic airway inflammation via activation of eosinophils and epithelial cells. Therefore, the regulation of EET formation or function may be a novel approach for the treatment of asthmatic patients.

\section{Acknowledgements}

This study was supported by a grant from the Korean Health Technology R \& D Project, Ministry of Health \& Welfare, Republic of Korea (HI14C2628).

\section{Authors' contributions}

Y.C. and L.D.P. designed the experiments, analyzed the data, and wrote the paper. D.H.L. performed the experiments. S.H.L. analyzed the clinical data. S.H.K. helped design the experiments. H.S.P. provided overall supervision for the entire study.

\section{Author details}

'Department of Allergy and Clinical Immunology, Ajou University School of Medicine, Suwon, South Korea. ${ }^{2}$ Faculty of Medicine, University of Medicine and Pharmacy, Ho Chi Minh, Vietnam. ${ }^{3}$ Clinical Trial Center, Ajou University Medical Center, Suwon, South Korea

\section{Conflict of interest}

The authors declare that they have no conflict of interest.

\section{Publisher's note}

Springer Nature remains neutral with regard to jurisdictional claims in published maps and institutional affiliations.

Supplementary information accompanies this paper at https://doi.org/ 10.1038/s12276-018-0136-8.

Received: 21 March 2018 Revised: 18 May 2018 Accepted: 28 May 2018. Published online: 16 August 2018

\section{References}

1. Lambrecht, B. N. \& Hammad, H. The immunology of asthma. Nat. Immunol. 16, 45-56 (2015).

2. Busse, W. W., Banks-Schlegel, S. \& Wenzel, S. E. Pathophysiology of severe asthma. J. Allergy Clin. Immunol. 106, 1033-1042 (2000). 
3. Fajt, M. L. \& Wenzel, S. E. Development of new therapies for severe asthma. Allergy Asthma Immunol. Res. 9, 3-14 (2017).

4. Macedo, P. et al. Inflammatory biomarkers in airways of patients with severe asthma compared with non-severe asthma. Clin. Exp. Allergy 39, 1668-1676 (2009).

5. Bousquet, J. et al. Eosinophilic inflammation in asthma. N. Engl. J. Med. $\mathbf{3 2 3}$ 1033-1039 (1990)

6. Schleich, F. N. et al. Importance of concomitant local and systemic eosinophilia in uncontrolled asthma. Eur. Respir. J. 44, 97-108 (2014).

7. Rothenberg, M. E. Eosinophilia. N. Engl. J. Med. 338, 1592-1600 (1998).

8. Holgate, S. T. The epidemic of allergy and asthma. Nature 402, B2-B4 (1999).

9. Green, R. H. et al. Asthma exacerbations and sputum eosinophil counts: a randomised controlled trial. Lancet 360, 1715-1721 (2002).

10. Jatakanon, A., Lim, S., Kharitonov, S. A., Chung, K. F. \& Barnes, P. J. Correlation between exhaled nitric oxide, sputum eosinophils, and methacholine responsiveness in patients with mild asthma. Thorax 53, 91-95 (1998).

11. Humbles, A. A. et al. A critical role for eosinophils in allergic airways remodeling. Science 305, 1776-1779 (2004).

12. Griffin, E. et al. Blood eosinophil number and activity in relation to lung function in patients with asthma and with eosinophilia. J. Allergy Clin. Immunol. 87, 548-557 (1991).

13. Sanz, M. L., Parra, A., Prieto, I., Dieguez, I. \& Oehling, A. K. Serum eosinophil peroxidase (EPO) levels in asthmatic patients. Allergy 52, 417-422 (1997).

14. Zheutlin, L. M., Ackerman, S. J., Gleich, G. J. \& Thomas, L. L. Stimulation of basophil and rat mast cell histamine release by eosinophil granule-derived cationic proteins. J. Immunol. 133, 2180-2185 (1984).

15. Yousefi, S. et al. Catapult-like release of mitochondrial DNA by eosinophils contributes to antibacterial defense. Nat. Med. 14, 949-953 (2008).

16. Dworski, R., Simon, H. U., Hoskins, A. \& Yousefi, S. Eosinophil and neutrophil extracellular DNA traps in human allergic asthmatic airways. J. Allergy Clin. Immunol. 127, 1260-1266 (2011).

17. Kerstan, A., Simon, H. U., Yousefi, S. \& Leverkus, M. Extensive accumulation of eosinophil extracellular traps in bullous delayed-pressure urticaria: a pathophysiological link? Br. J. Dermatol. 166, 1151-1152 (2012).

18. Gevaert, E. et al. Extracellular eosinophilic traps in association with Staphylococcus aureus at the site of epithelial barrier defects in patients with severe airway inflammation. J. Allergy Clin. Immunol. 139, 1849-1860 e1846 (2017).

19. Ueki, S. et al. Eosinophil extracellular DNA trap cell death mediates lytic release of free secretion-competent eosinophil granules in humans. Blood 121, 2074-2083 (2013)
20. Yousefi, S., Simon, D. \& Simon, H. U. Eosinophil extracellular DNA traps: molecular mechanisms and potential roles in disease. Curr. Opin. Immunol. 24 736-739 (2012).

21. Ueki, S. et al. Eosinophil extracellular trap cell death-derived DNA traps: their presence in secretions and functional attributes. J. Allergy Clin. Immunol. 137, 258-267 (2016).

22. Fuchs, T. A. et al. Novel cell death program leads to neutrophil extracellular traps. J. Cell Biol. 176, 231-241 (2007).

23. Papayannopoulos, V., Metzler, K. D., Hakkim, A. \& Zychlinsky, A. Neutrophil elastase and myeloperoxidase regulate the formation of neutrophil extracellular traps. J. Cell Biol. 191, 677-691 (2010).

24. Cheng, O. Z. \& Palaniyar, N. NET balancing: a problem in inflammatory lung diseases. Front Immunol. 4, 1 (2013).

25. Fuchs, T. A. et al. Extracellular DNA traps promote thrombosis. Proc. Natl Acad Sci. USA 107, 15880-15885 (2010).

26. Papayannopoulos, V. Neutrophil extracellular traps in immunity and disease. Nat. Rev. Immunol. 18, 134-147 (2018).

27. Choi, Y. et al. Neutrophil extracellular DNA traps induce autoantigen production by aimay epithelial cells. Mediat. Inflamm. 2017, 5675029 (2017).

28. Hwang, E. K. et al. The predictors of poorly controlled asthma in elderly. Allergy Asthma Immunol. Res. 4, 270-276 (2012).

29. Chung, K. F. et al. International ERS/ATS guidelines on definition, evaluation and treatment of severe asthma. Eur. Respir. J. 43, 343-373 (2014).

30. Lambrecht, B. N. \& Hammad, H. The airway epithelium in asthma. Nat. Med. 18, 684-692 (2012).

31. Schleimer, R. P., Kato, A., Kern, R., Kuperman, D. \& Avila, P. C. Epithelium: at the interface of innate and adaptive immune responses. J. Allergy Clin. Immunol. 120, 1279-1284 (2007).

32. White, S. R. Apoptosis and the airway epithelium. J. Allergy 2011, 948406 (2011).

33. Akdis, M. et al. Interleukins (from IL-1 to IL-38), interferons, transforming growth factor beta, and TNF-alpha: receptors, functions, and roles in diseases. J. Allergy Clin. Immunol. 138, 984-1010 (2016).

34. Shichijo, K. et al. Antibody to eosinophil cationic protein suppresses dextran sulfate sodium-induced colitis in rats. World J. Gastroenterol. 11, 4505-4510 (2005).

35. Bradding, P., Walls, A. F. \& Holgate, S. T. The role of the mast cell in the pathophysiology of asthma. J. Allergy Clin. Immunol. 117, 1277-1284 (2006).

36. Cunha, A. A. et al. Extracellular DNA traps in bronchoalveolar fluid from a murine eosinophilic pulmonary response. Allergy 69, 1696-1700 (2014). 